\title{
H2A.B facilitates transcription elongation at methylated CpG loci
}

\author{
Yibin Chen, ${ }^{1}$ Qiang Chen, ${ }^{1}$ Richard C. McEachin, ${ }^{2}$ James D. Cavalcoli, ${ }^{2}$ \\ and Xiaochun $\mathrm{Yu}^{1,3}$ \\ ${ }^{1}$ Division of Molecular Medicine and Genetics, Department of Internal Medicine, ${ }^{2}$ Department of Computational \\ Medicine and Bioinformatics, University of Michigan Medical School, Ann Arbor, Michigan 48109, USA
}

\begin{abstract}
H2A.B is a unique histone H2A variant that only exists in mammals. Here we found that H2A.B is ubiquitously expressed in major organs. Genome-wide analysis of H2A.B in mouse ES cells shows that H2A.B is associated with methylated DNA in gene body regions. Moreover, H2A.B-enriched gene loci are actively transcribed. One typical example is that H2A.B is enriched in a set of differentially methylated regions at imprinted loci and facilitates transcription elongation. These results suggest that $\mathrm{H} 2 \mathrm{~A}$.B positively regulates transcription elongation by overcoming DNA methylation in the transcribed region. It provides a novel mechanism by which transcription is regulated at DNA hypermethylated regions.
\end{abstract}

[Supplemental material is available for this article.]

Histones are nuclear proteins in eukaryotes that package genomic DNA into structural units called nucleosomes (Andrews and Luger 2011). A nucleosome consists of a histone octamer with two copies each of four core histones that are wrapped with $\sim 146 \mathrm{bp}$ of double-stranded DNA (Luger et al. 1997; Andrews and Luger 2011). This basic unit allows histones to regulate most DNA-dependent biological processes, such as gene transcription (Berger 2002; Campos and Reinberg 2009).

Besides the four canonical core histones, several other histone variants have emerged during evolution, such as $\mathrm{H} 2 \mathrm{AX}, \mathrm{H} 2 \mathrm{AZ}$, macroH2A, and H3.3. These histone variants are also incorporated into the chromatin and are usually located at special regions to provide unique regulation of DNA-dependent biological processes. Among these histone variants, H2A.B (histone H2A-Barr body deficient, also named H2A.Bbd) is the newest histone variant in evolution and only exists in mammals with unique biochemical properties (Chadwick and Willard 2001; Eirin-Lopez et al. 2008). H2A.B is encoded by three copies of the $H 2 A F B$ gene at the subtelomere region of the $\mathrm{X}$ chromosomes in both mice and humans. In mice, H2A.B1 and H2A.B2 (encoded by H2afb3 and H2afb2, respectively) are actively transcribed (Ishibashi et al. 2010). H2A.B is a unique histone $\mathrm{H} 2 \mathrm{~A}$ variant that shares only $40 \%-50 \%$ sequence identity with canonical H2A (Chadwick and Willard 2001). It has quickly evolved with remarkable sequence diversities among different species. In vitro analysis suggests that H2A.B can replace canonical $\mathrm{H} 2 \mathrm{~A}$ in the nucleosome. In comparison to canonical $\mathrm{H} 2 \mathrm{~A}$, H2A.B neither has the key residues to form the "acid patch" in the nucleosome, nor does it contain the C-terminal tail that is usually ubiquitinated in canonical $\mathrm{H} 2 \mathrm{~A}$ and other variants of $\mathrm{H} 2 \mathrm{~A}$ (Zhou et al. 2007; Gonzalez-Romero et al. 2008). Moreover, a histone octamer containing H2A.B is merely wrapped by $118-130$ DNA base pairs compared to the $146 \mathrm{bp}$ associated with the canonical histone octamer (Bao et al. 2004; Doyen et al. 2006). The unusual biochemical characteristics of H2A.B raise the possibility that H2A.B may play an important role in regulating biological events in the nucleus, such as transcription (Angelov et al. 2004; Gautier et al.

\footnotetext{
${ }^{3}$ Corresponding author

E-mail xiayu@umich.edu

Article published online before print. Article, supplemental material, and publication date are at http://www.genome.org/cgi/doi/10.1101/gr.156877.113.
}

2004; Eirin-Lopez et al. 2008; Ishibashi et al. 2010; Soboleva et al. 2012; Tolstorukov et al. 2012).

Although H2A.B has been well characterized in vitro, the function and localization of H2A.B in the genome are still unclear. In this study, using whole-genome deep sequencing, we found that H2A.B is mainly incorporated into gene body regions and associated with DNA methylation. Knockdown of H2A.B reduces the transcription of a set of H2A.B-bound genes. Particularly at some imprinting loci, where H2A.B is only incorporated in differentially methylated regions (DMR), depletion of H2A.B only reduces the transcription of methylated alleles. Collectively, our data elicit a novel function of H2A.B: that it regulates gene transcription at DNA methylated gene body regions.

\section{Results}

H2A.B is associated with $5 \mathrm{mC}$ in the gene body regions

In order to study the function of H2A.B in vivo, we first generated antibodies against H2A.B1 and H2A.B2 (anti-H2A.B1 and antiH2A.B2 antibodies) (Supplemental Fig. S1A,B), as the primary sequences of H2A.B1 and H2A.B2 are slightly different at the $\mathrm{N}$ terminus. We also generated an antibody against the common C-terminal regions in both H2A.B1 and H2A.B2 (anti-H2A.B antibody). This antibody recognizes both H2AB.1 and H2A.B2 (Supplemental Fig. S1B-D).

Although the mRNA level and protein level of both H2A.B1 and H2A.B2 are higher in testis (Supplemental Fig. S1E; Ishibashi et al. 2010), comparable H2A.B1 and H2A.B2 were detected in mouse ES cells and other major organs (Fig. 1A). Moreover, although $H 2 a f b 3$ and $H 2 a f b 2$ localize to the X chromosome, we did not observe any gender difference in the expression of H2A.B (Supplemental Fig. S1F). Thus, these results suggest that H2A.B is ubiquitously expressed and may participate in major biological processes. To study the distribution of H2A.B on chromatin, we 
A

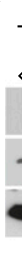

Female

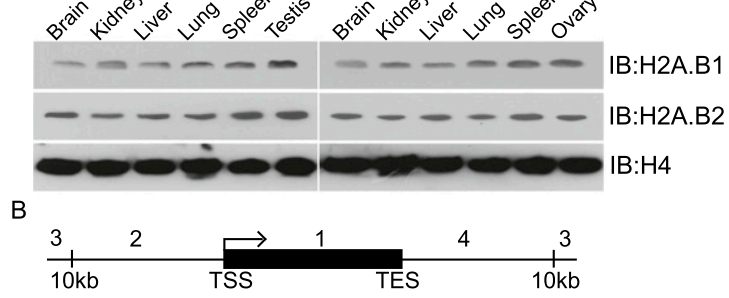

Genomic distribution of H2A.B-enriched regions

intergenic region: $43.2 \%$

2 upsteam (0-10 kb): $10.9 \%$

3 distal intergenic: $21.8 \%$

4 downstream $(0-10 \mathrm{~kb}): 10.5 \%$

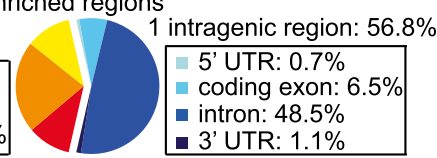

a intron: $48.5 \%$
C

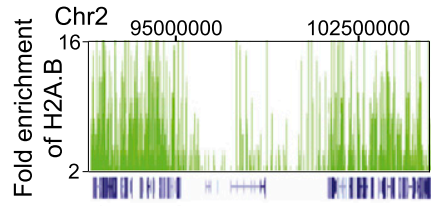

D

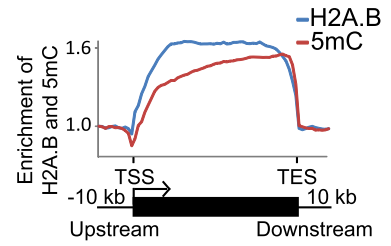

E

H2A.B
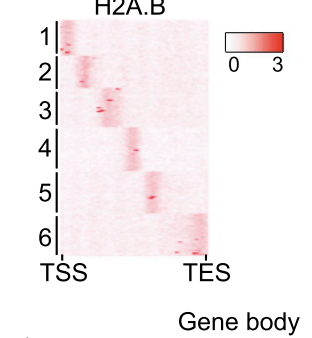
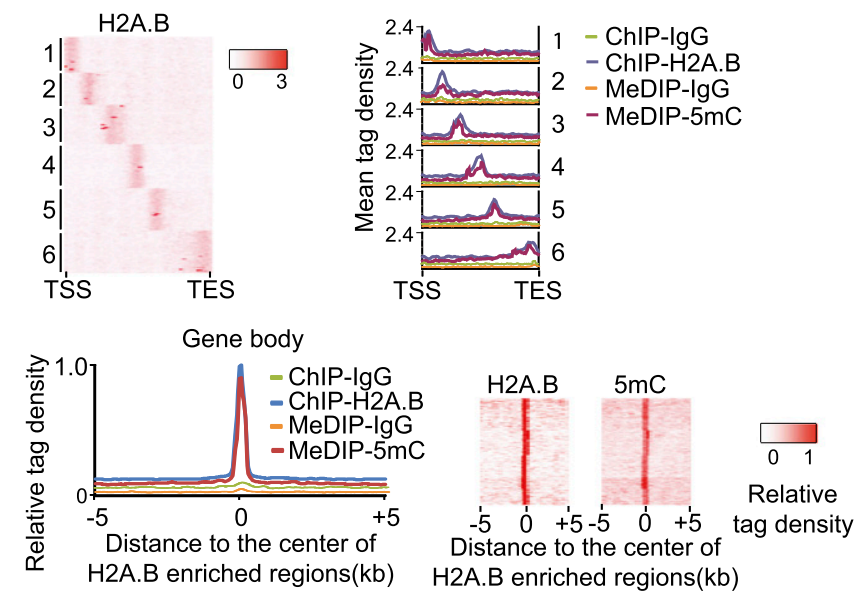

G
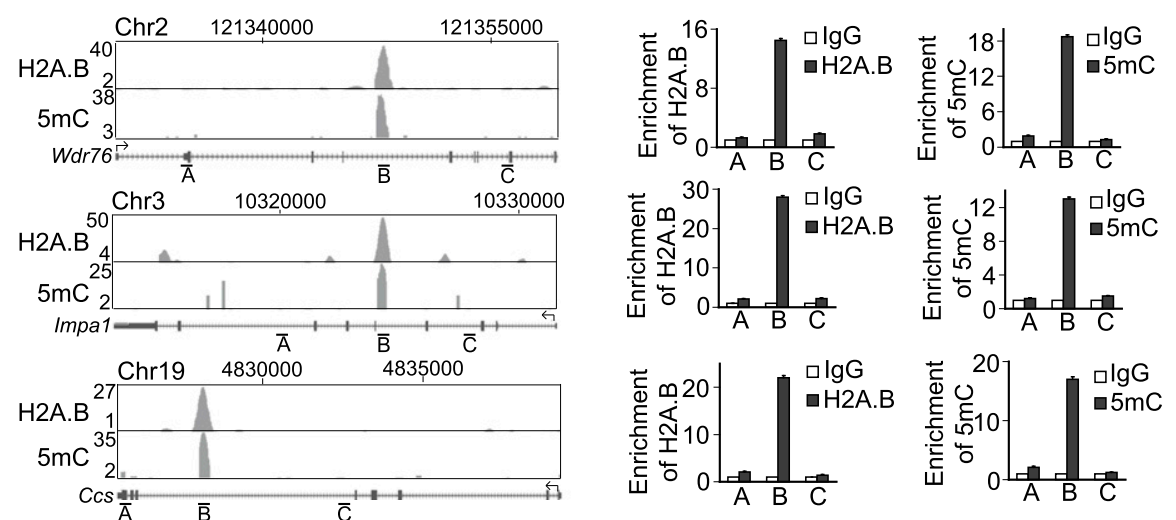

Figure 1. H2A.B is associated with $5 \mathrm{mC}$ in the gene body. $(A)$ H2A.B is ubiquitously expressed in major organs. Chromatin fractions from different mouse tissues are subjected to Western blotting with anti-H2A.B1 and H2A.B2 antibodies. Anti-histone H4 is used as a protein loading control. (B) Genomic distribution of H2A.B in different regions is summarized in a pie chart. (TSS) transcription start site, (TES) transcription end site. (C) A snapshot of the distribution of H2A.B (green) in the region of chr2: 90,353,915-106,163,465 in mouse ES cells. Gene structure is indicated in blue at the bottom of the plot. ( $X$-axis) The indicated genomic region; ( $y$-axis) the fold enrichment of $\mathrm{H} 2 \mathrm{~A}$.B compared to the irrelevant lgG control. ( $D$ ) Relative enrichment of H2A.B and $5 \mathrm{mC}$ within $10 \mathrm{~kb}$ upstream of and downstream from the gene body in mouse ES cells. ( $X$-axis) Relative gene position; ( $y$-axis) relative fold enrichment. $(E)$ Heat map represents the clusters of H2A.B enrichment in gene bodies in mouse ES cells. The tag density of H2A.B is calculated and subjected to k-means clustering. Six major clusters were indicated (left panel). Mean tag density of $\mathrm{H} 2 \mathrm{~A}$. B and $5 \mathrm{mC}$ are plotted in each cluster (right panel). ( $Y$-axes) Mean tag density. Irrelevant lgG was used as the control in ChIP-seq and MeDIP-seq. $(F)$ Normalized tag density of 5 mC in H2A.B-enriched gene body region. The detailed analyses are included in the Methods section. $(G)$ Representative regions show the overlap between $\mathrm{H} 2 \mathrm{~A}$. $\mathrm{B}$ and $5 \mathrm{mC}$. In the left panels, the $x$-axes indicate the genomic regions. The $y$-axes represent the fold enrichment of H2A.B and $5 \mathrm{mC}$. Three different regions in each gene are examined by ChIP-qPCR and MeDIP-qPCR to confirm the ChIP-seq and MeDIP-seq analyses. Data are presented as mean \pm SEM ( $n=3$ ).

performed genome-wide chromatin IP-sequencing (ChIP-seq) analysis using anti-H2A.B antibodies in mouse ES cells. A total of 26,741 genomic regions enriched with H2A.B were identified with high confidence (Supplemental Figs. S2, S3). The whole genome was partitioned into four regions: intragenic region; upstream region (10 kb upstream of TSS, including most transcription promoters and other transcriptional elements); distal intergenic region that does not encode any genes; and downstream region (10 
$\mathrm{kb}$ downstream from TES). Interestingly, $\sim 57 \%$ of H2A.B-enriched areas were found in the intragenic region (Fig. 1B,C). Among the intragenic regions, most H2A.B is associated with coding exons and introns (Fig. 1B), suggesting that H2A.B is enriched in the gene body region (Fig. 1D).

We noticed that DNA methylation is also associated with the gene body region (Haines et al. 2001; Burden et al. 2005; Grandjean et al. 2007; Pastor et al. 2011). To examine if there is any correlation between H2A.B and DNA methylation, we first performed methylated DNA immunoprecipitation (MeDIP) to validate the profile of $5 \mathrm{mC}$ in mouse ES cells. Consistent with other published results (Dawson and Trapp 2004; Wu et al. 2011), $5 \mathrm{mC}$ is also enriched in gene bodies (Fig. 1D; Supplemental Fig. S4A). We compared the H2A.B- and 5mC-enriched regions and found that $\sim 51 \%$ of H2A.Benriched regions overlapped with $5 \mathrm{mC}$ enriched regions in the mouse genome (Supplemental Fig. S4B). Since both H2A.B and $5 \mathrm{mC}$ are enriched in gene body regions, we examined H2A.B and $5 \mathrm{mC}$ overlapped gene bodies. H2A.B-enriched regions inside of gene bodies are further clustered into six groups. We found that each of those enriched region groups is associated with $5 \mathrm{mC}$ (Fig. 1E). Further analyses across the H2A.B enrichment regions show that the $5 \mathrm{mC}$ profile is also associated with the H2A.B profile in gene bodies (Fig. 1F). Moreover, we randomly picked three H2A.B-enriched regions and performed ChIP assays to confirm the high-throughput ChIP-seq results (Fig. 1G).

Thus, this accumulated evidence suggests that H2A.B is located in gene bodies and clearly associated with $5 \mathrm{mC}$.

In mammals, most $5 \mathrm{mC}$ exists in the $\mathrm{CpG}$ dinucleotide context (Yan et al. 2011; Ziller et al. 2011; Kim et al. 2013). It has been reported that $60 \%-90 \%$ of $\mathrm{CpG}$ dinucleotide is methylated in mammals (Ehrlich et al. 1982; Tucker 2001). Like 5mC, methylated $\mathrm{CpG}$ is also enriched in gene body regions (Supplemental Fig. S4A). Since each CpG has a different level of methylation, we examined and found that H2A.B-bound gene body regions are mainly associated with CpG with a high level of methylation (Supplemental Fig. S4C). Moreover, the density of CpG with a high level of methylation in H2A.B-bound gene body regions is much higher than that in H2A.B-unbound gene body regions (Supplemental Fig. S4D). With this evidence taken together, we demonstrate that H2A.B is associated with DNA methylation in the gene body regions.

\section{H2A.B regulates gene transcription}

Since H2A.B is enriched in the gene body regions, we asked if H2A.B is involved in gene transcription. We examined global gene transcription levels in mouse ES cells and found that the average expression level of H2A.B-bound genes is higher than that of H2A.B-unbound genes (Fig. 2A), suggesting that H2A.B is likely
C

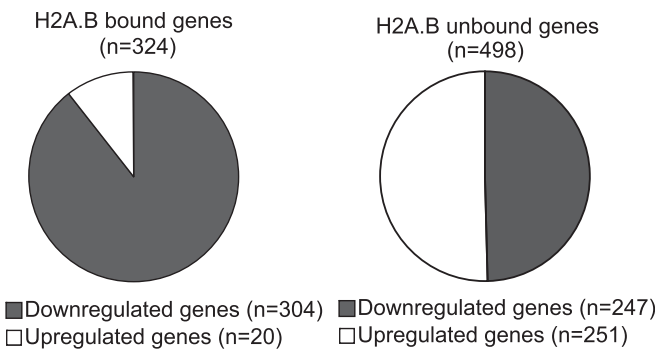

D

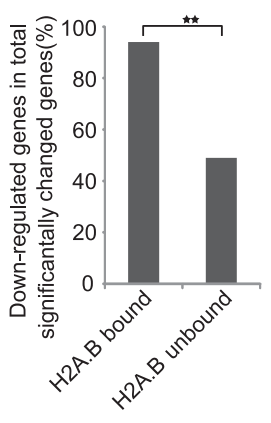

to facilitate gene transcription. Next, we decreased the expression of H2A.B1 and H2A.B2 in mouse ES cells using shRNA (Supplemental Fig. S1D) and found that the transcription of H2A.B-occupied genes was significantly reduced (Fig. 2B). Among H2A.B-occupied genes, we found that the transcription of 324 genes was significantly changed (by a more than twofold difference) when H2A.B was depleted (Fig. 2C). Among these 324 genes, transcription of $\sim 94 \%$ genes was down-regulated, indicating that H2A.B is likely to facilitate gene transcription (Fig. 2D). We randomly selected five H2A.B-bound genes with significant down-regulation and confirmed the transcription status using RT-qPCR (Supplemental Fig. S5A). We also examined the H2A.B-unbound genes. The transcription of 498 H2A.B-unbound genes was significantly altered (by a more than twofold difference) (Fig. 2C). In contrast, only $51 \%$ of H2A.B-unbound genes was significantly down-regulated (Fig. 2D), which is likely to be caused by indirect or secondary effects from H2A.B depletion. Moreover, the $5 \mathrm{mC}$ level in the downregulated H2A.B-bound genes was significantly higher than that in the unchanged or up-regulated H2A.B-bound genes, whereas little difference in DNA methylation level was observed in each subgroup of H2A.B-unbound genes (Supplemental Fig. S5B).

\section{Genome Research} www.genome.org 
H2A.B regulates gene transcription at imprinting loci

We further examined the most downregulated genes in the absence of H2A.B and found a unique phenomenon in that H2A.B is associated with the expression of several imprinted genes (Supplemental Table S1). The majority of imprinted genes in mammals has been found to have roles in embryonic growth and development, including development of the placentas, which only exists in mammals. Interestingly, this set of imprinted genes is methylated in the gene body region in one allele, while the methylated allele is still actively transcribed. One typical example is the Kcnq1 locus. At this locus, the maternal allele is still actively transcribed even with the differentially methylated region (DMR) in the gene body. However, the paternal allele is silenced by the expression of the noncoding RNA Kcnq1ot1 (Lee et al. 1999; Mitsuya et al. 1999; Ramsahoye et al. 2000; Thakur et al. 2004; Kozlenkov et al. 2013). To carefully examine the biological function of H2A.B in transcriptional regulation, we generated a hybrid ES cell line with maternal chromosomes derived from $M . m$. musculus (mus) and paternal chromosomes from M. m. castaneus (cas). It has been reported that the mouse Kcnq1 gene harbors a methylated DMR at intron 10 in the maternal allele during oocytogenesis (Weaver et al. 2009), where H2A.B is highly enriched (Fig. 3A). To further confirm the specific incorporation of H2A.B in Kcnq1, five different regions of this imprinted locus, including the DMR, were probed (Fig. 3B). ChIP analysis revealed that H2A.B was exclusively enriched in one region corresponding to the DMR but not other adjacent regions (Fig. 3C). Since ChIP analysis could not distinguish the DMR in the maternal allele and the unmethylated paternal allele, we took two different approaches to distinguish maternal and paternal alleles. First, the H2A.B-associated DNA fragments were sequenced. Based on single nucleotide polymorphisms (SNPs), we found that H2A.B was only deposited in the maternal DMR instead of the unmethylated paternal allele (Fig. 3D). Second, the H2A.B-associated DNA fragments were digested by the selective restriction enzyme BmgBI. Due to the presence of SNPs, the DNA fragments from maternal and paternal alleles were digested into different patterns. Again, H2A.B was clearly shown to be only deposited in the maternal DMR (Fig. $3 \mathrm{E})$. These results demonstrate that H2A.B is mainly localized at the maternal DMR of Kcnq1. To examine the role of H2A.B in the transcription of Kcnq1, we used two different shRNAs to stably

B

C

G and cas.
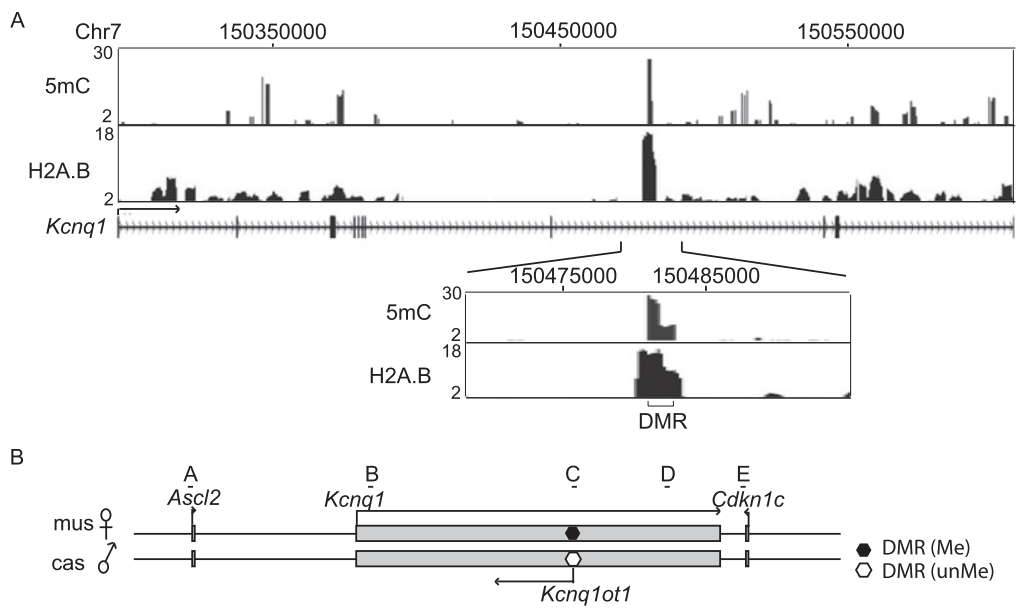

D
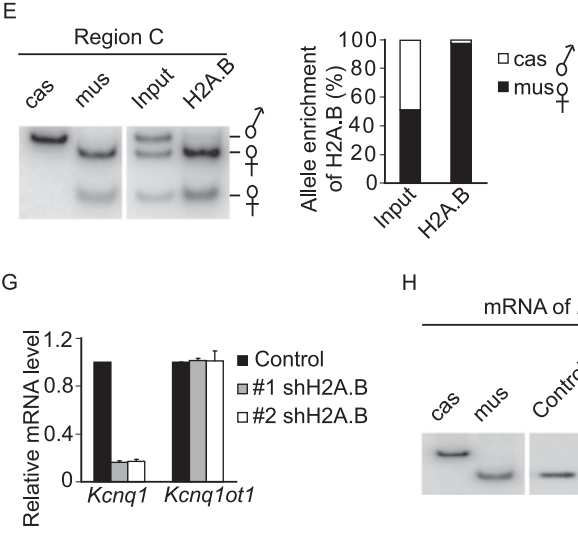

$\mathrm{H}$
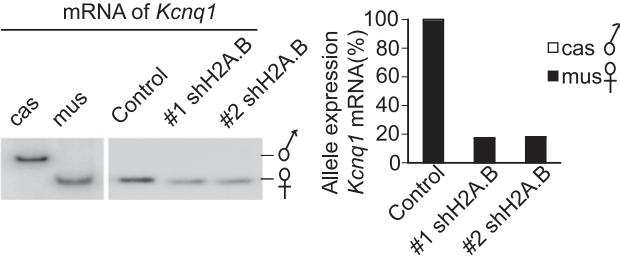

Figure 3. H2A.B regulates the transcription of the imprinted Kcnq1 locus. $(A)$ ChIP-seq and MeDIPseq profile of H2A.B and $5 \mathrm{mC}$ in Kcnq 1 loci. The $x$-axes indicate the analyzed genomic region. The $y$-axes represent the fold enrichment of $5 \mathrm{mC}$ or $\mathrm{H} 2 \mathrm{~A}$.B compared to the irrelevant lgG control. Enlarged view shows the colocalization of H2A.B and $5 \mathrm{mC}$ in the DMR region. (B) Schematic sketch of the Kcnq1 locus. (C) ChIP analysis confirms that H2A.B is incorporated into the DMR of the Kcnq1 locus but not other regions. Data are presented as mean $\pm \operatorname{SEM}(n=4) .(D, E)$ H2A.B is enriched in the maternal DMR of Kcnq1. ChIP assays are performed using anti-H2A.B antibody. Based on the SNPs between M. $m$. musculus (mus) and M. m. castaneus (cas), allele-specific deposition of H2A.B is determined by DNA sequencing $(D)$ or the BmgBI digestion of an amplified DNA fragment into different patterns $(E)$. $(F)$ shRNA treatment depletes H2A.B at the DMR of Kcnq1. ChIP analyses of H2A.B at the DMR and the adjacent region are performed. Data are presented as mean \pm SEM $(n=4)$. (G) Knockdown of H2A.B suppresses the transcription of the Kcnq1 gene. Data are presented as mean \pm SEM $(n=4)$. $(H)$ The transcription of Kcnq1 is dependent on the maternal allele and H2A.B. Allele-specific expression of imprinted Kcnq1 was determined by different Nlalll digestion patterns based on the SNP between mus

knock down both H2A.B1 and H2AB.2 in the mus/cas ES cells (Supplemental Fig. S6A). As shown in Figure 3F, the incorporation of H2A.B at Kcnq1 loci was significantly reduced in the ES cells following the stable knockdown of H2A.B. Correspondingly, the transcription of Kcnq1, but not the transcription of adjacent genes such as $A s c l 2$ and $C d k n 1 c$, was significantly suppressed (Fig. 3G; Supplemental Fig. S6B-D). The transcription of Kcnq1ot1 was not induced in the maternal allele either (Fig. 3G; Supplemental 
Fig. S6E). Of note, the transcription of Kcnq1 was only from the maternal allele since Kcnq1ot1, transcribed from the paternal allele in an antisense orientation, suppresses the transcription of Kcnq1 in the paternal allele (Mancini-Dinardo et al. 2006; Green et al. 2007). Thus, the downregulation of maternally transcribed Kcnq1 reduces the overall mRNA level of Kcnq1 (Fig. 3H).

To further validate that H2A.B facilitates the transcription of imprinted genes, we also examined the transcription of $I g f r$. Like $K c n q 1$, the $I g f r$ gene also contains a maternal DMR in the gene body region (Fig. 4A). Different from the Kcnq1 locus, imprinting of the $I g f 2 r$ locus is established during implantation (Risinger et al. 2003; Latos et al. 2009). Thus, we could not detect obvious H2A.B deposition in undifferentiated ES cells. However, when ES cells were treated with retinoic acid (RA) to induce differentiation, the maternal imprinting of $I g f 2 r$ was established during the ES cell differentiation (Supplemental Fig. S7A; Latos et al. 2009). Simultaneously, deposition of H2A.B into the methylated DMR but not other unmethylated regions at the Igf2r locus was observed using ChIP and DNA sequencing (Fig. 4B,C). The results again were confirmed by PCR-based single-strand conformation polymorphism (PCR-SSCP) (Fig. 4D). Along with the establishment of imprinting, the expression of $I g f r$ was dramatically increased during ES cell differentiation that is indicated by other ES cell differentiation makers, such as the down-regulation of Pou5f1 and up-regulation of Fgf5 (Supplemental Fig. S7B,C). Like the expression of Kcnq1, Igf $2 r$ mRNA is mainly transcribed from the imprinted maternal allele, since a noncoding RNA Airn, transcribed from the unmethylated paternal allele in the antisense orientation, suppresses the transcription of $I g f 2 r$ in the paternal allele (McCormick et al. 2001; Braidotti et al. 2004). Knockdown of H2A.B at the $I g f 2 r$ locus significantly suppressed the transcription of $I g f r$ from the methylated maternal allele but did not activate the transcription of $I g f r$ from the unmethylated paternal allele after RA induction (Fig. 4E-G) nor the transcription of the adjacent gene Slc22a2 (Supplemental Fig. S7D). Taken together, these results demonstrate that H2A.B is important for the transcription of imprinted alleles.

H2A.B facilitates transcription elongation at the methylated DMR

Since H2A.B is not enriched at the TSS of target genes, the accumulation of RNA polymerase II (Pol II), the transcription ma-

B

D

$\mathrm{F}$
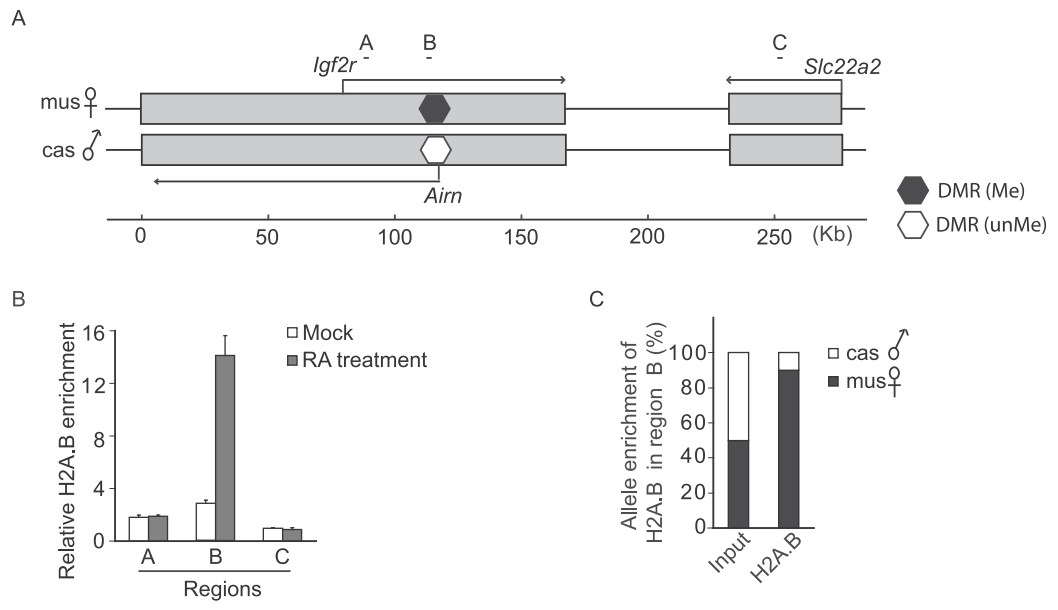

C
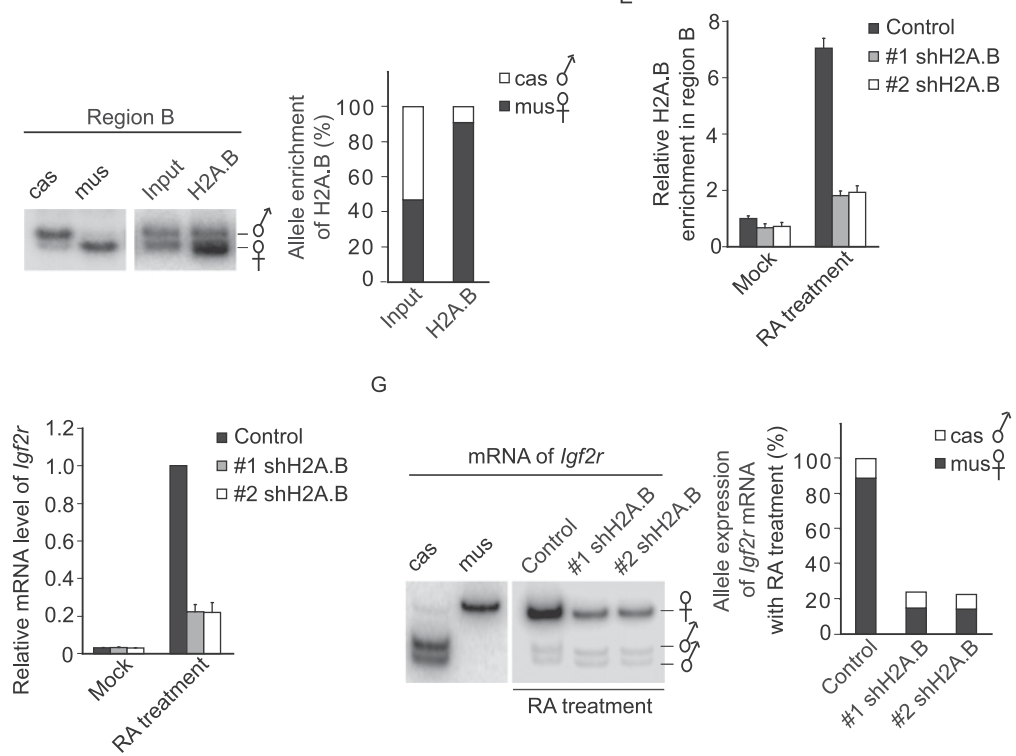

Figure 4. H2A.B regulates the transcription of the imprinted lgf2r locus. $(A)$ Schematic sketch of the Igf2r locus. (B) Retinoic acid (RA) induces the enrichment of H2A.B at the Igf2r locus. ChIP analysis on the $\lg 2 \mathrm{r}$ locus is performed in mouse ES cells with or without RA treatment. Data are presented as mean $\pm \operatorname{SEM}(n=3)$. $(C, D)$ H2A.B is deposited at the maternally methylated DMR of Igf2 $r$ following RA treatment. Allele-specific incorporation of H2A.B was determined by DNA sequencing $(C)$ or PCR-SSCP $(D)$ (see Methods). (E) Knockdown of H2A.B suppresses the incorporation of H2A.B at the DMR of $l g f 2 r$. ChIP analysis is performed in shRNA-treated ES cells with or without RA induction. Data are presented as mean \pm SEM $(n=3)$. ( $F$ ) Knockdown of H2A.B suppresses the RA-induced transcription of lgf $2 r$. The relative mRNA level of $\lg 2 r$ is examined by qPCR. Data are presented as mean $\pm \operatorname{SEM}(n=4)$. (G) Knockdown of H2A.B suppresses the transcription of Igf $2 r$ from the maternal allele following RA treatment. Left panel shows that maternal and paternal alleles are digested by Taql into different patterns because of SNP. Right panel shows that the transcription of $\lg f 2 r$ is dependent on the maternally methylated allele following RA treatment, and that depletion of H2A.B suppresses the RA-induced transcription of $\lg f 2 \mathrm{r}$ from the maternal allele. The reduction of the transcription of $I g f 2 r$ from each allele following RA treatment is summarized in the histogram.

chinery, was not changed at the TSS of Kcnq1 and Igf $2 r$ when H2A.B was down-regulated (Fig. 5A), suggesting that H2A.B might not regulate transcription initiation. Since H2A.B is enriched at the gene body regions of $K c n q 1$ and $I g f r$, we asked if H2A.B regulates transcription elongation. Ser2 phosphorylation of the C-terminal domain of Pol II is the surrogate marker of Pol II during transcription elongation (Phatnani and Greenleaf 2006). Thus, we examined the status of Ser2 phosphorylated Pol II in both Kcnq1 and $I g f 2 r$ loci. Interestingly, down-regulation of H2A.B arrested

\section{Genome Research www.genome.org}


A

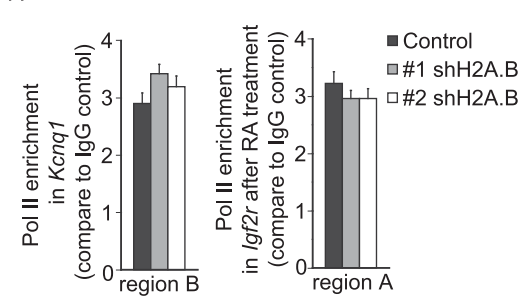

C
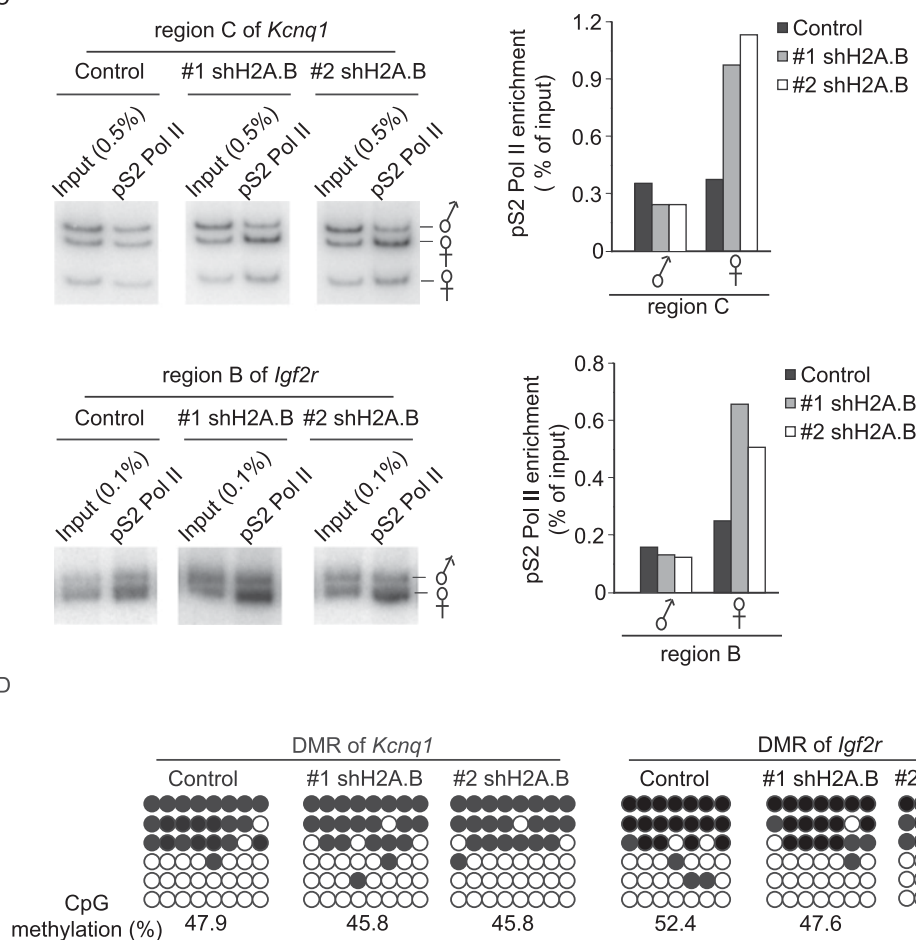

DMR of $\lg f 2 r$

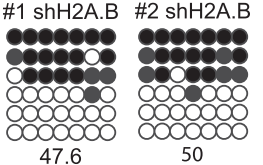

Figure 5. H2A.B facilitates transcription elongation at the methylated DMR. (A) Down-regulation of H2A.B does not affect the Pol Il deposition at the TSS of Kcnq1 and Igf2r genes. ChIP analyses in the TSS regions (region $\mathrm{B}$ of the Kcnq1 gene and region $\mathrm{A}$ of the Igf2r gene) are performed using an anti-RNA Pol II antibody. Data are presented as mean \pm SEM $(n=3)$. (B) Down-regulation of H2A.B arrests the pSer2 Pol II at the DMR of Kcnq1 and Igf2r. ChIP analyses at the DMR regions (region C of the Kcnq1 gene and region B of the Igf2r gene) are performed using an anti-RNA Pol II pSer2-CTD antibody. Data are presented as mean \pm SEM $(n=3)$. (C) Based on the SNPs between mus and cas, allele-specific deposition of pSer2 Pol II in Kcnq1 and Igf2r is determined by restriction enzyme BmgBI digestion or SSCP. (D) Down-regulation of H2A.B by shRNA does not affect the DNA methylation status at the DMR of Kcnq1; down-regulation of H2A.B by shRNA does not affect the DNA methylation status at the DMR of Igf2r after RA treatment.

Ser2-phosphorylated Pol II at the DMR of both the Kcnq1 and Igf $2 r$ loci (Fig. 5B). To examine whether Ser2-phosphorylated Pol II is specifically arrested at the DMR of the maternal allele but not the unmethylated paternal allele, Ser2-phosphorylated Pol II-associated DNA fragments were digested by BmgBI. Due to the presence of SNP, the DNA fragments from maternal and paternal alleles were digested into different patterns. We found that down-regulation of H2A.B induced Ser2-phosphorylated Pol II accumulation at the methylated DMR of Kcnq1. We also performed PCR-SSCP to distinguish the accumulation of Ser2-phosphorylated Pol II in maternal and paternal alleles $I g f r$ locus. Again, based on the SNP, we found that Ser2-phosphorylated Pol II is mainly associated with the methylated DMR of the maternal allele at the Igf $2 r$ locus (Fig. 5C), suggesting that loss of H2A.B impairs Pol II-dependent ent regions were probed (Supplemental sistent with ChIP-seq and MeDIP-seq results, H2A.B and $5 \mathrm{mC}$ were enriched in region $\mathrm{C}$ of the Stau2 gene (Supplemental Fig. S9B,C). Loss of H2A.B did not affect the DNA methylation status (Supplemental Fig. S9C), but significantly downregulated the transcription of Stau2 and induced Pol II accumulation (Supplemental Fig. S9D,E). Moreover, we found that loss of H2A.B up-regulated the transcription in 20 H2A.B-bound genes. We validated the results by examining one of these 20 genes, $D k k 3$. Although H2A.B is distributed in the Dkk3 gene (Supplemental Fig. S10A), loss of H2A.B up-regulated the transcription of the Dkk3 gene (Supplemental Fig. S10B,C). It is possible that up-regulation of a small group of H2A.B-bound genes like $D k k 3$ is indirectly mediated by the transcriptional down-regulation of many other H2A.B-bound genes. 


\section{Discussion}

Compared with canonical H2A, H2A.B lacks several important features. For example, it does not have the key residues to form the "acid patch" in the nucleosome, which abolishes the inter-nucleosome interaction (Bao et al. 2004; Doyen et al. 2006; Zhou et al. 2007). It does not contain the C-terminal tail, which is important for the interaction with histone $\mathrm{H} 3$ inside of the nucleosome and maintains the stability of the histone octamer (Ausio 2006; Gonzalez-Romero et al. 2008). The unique characteristics of H2A.B cause the nucleosome to become less stable, which could be the major reason that it facilitates transcription elongation through the methylated regions (Supplemental Fig. S11). Moreover, since H2A.B lacks a lysine residue at its $\mathrm{C}$ terminus, it cannot be ubiquitinated. Canonical H2A is ubiquitinated at the K119 site, which is a transcription repression mark (Cao et al. 2005). Thus, loss of modification by ubiquitin in the H2A.B removes the transcription repression mark and may positively regulate transcription.

Using several specific antibodies against H2A.B, we found that H2A.B is ubiquitously expressed in major organs, suggesting that H2A.B has a more important function than previously expected (Ishibashi et al. 2010; Soboleva et al. 2012). Interestingly, with different approaches, we found that H2A.B is deposited in hypermethylated gene body regions and facilitates the transcription elongation at methylated regions. Methylated DNA is thought to be recognized by methyl-CpG-binding domain proteins (MBD), which recruits other effectors to the chromatin, such as histone deacetylases and other chromatin remodelers, to silence gene transcription by forming compact and inactive chromatin, termed heterochromatin (Grewal and Moazed 2003; Klenov and Gvozdev 2005). Since H2A.B mainly exists in gene body regions, where gene transcription is not suppressed by gene body methylation (Jones 2012), it is possible that H2A.B relaxes the chromatin in the methylated gene body regions and facilitates transcription elongation, especially in the DMR of mammalian imprinted genes. Moreover, H2A.B is reported to be absent in the inactive $\mathrm{X}$ chromosome (Chadwick and Willard 2001). Interestingly, gene body methylation in the active $\mathrm{X}$ chromosome is significantly higher than that in the inactive $\mathrm{X}$ chromosome (Hellman and Chess 2007), which is also transcriptionally silenced. It is consistent with our finding that H2A.B is associated with $5 \mathrm{mC}$ in the gene body and facilitates transcription elongation. In our study, we found that H2A.B is not generally enriched at TSSs, suggesting that H2A.B may not regulate transcription initiation. Instead, several other histone variants, such as H2A.Z and H3.3, as well as specific histone modifications, are enriched at TSSs, which facilitates transcription initiation (Schwartz and Ahmad 2005; Anamika et al. 2010; Wang et al. 2013). In contrast, DNA methylation at the TSS is associated with transcription suppression because DNA methylation is likely to set the TSS in a compact stage to abrogate transcription initiation (Ehrlich et al. 1982; Shin et al. 2012; Wang et al. 2013).

In addition, besides H2A.B, H3.3 is another unique histone variant that is associated with active gene expression. Like H2A.B, H3.3 is incorporated in gene bodies and is enriched in the actively transcribed genes (Ahmad and Henikoff 2002; Schwartz and Ahmad 2005; Hodl and Basler 2009; Jullien et al. 2012). Moreover, the density of H3.3 over genes correlates with that of Pol II (Mito et al. 2005; Daury et al. 2006), suggesting that like H2A.B, H3.3 also regulates gene transcription. Thus, it is possible that H2A.B and H3.3 function together or in parallel to facilitate gene transcription. Future analysis of the overlapped regions of H2A.B and H3.3 may reveal the crosstalk between these histone variants in transcriptional regulation.

\section{Methods}

\section{Cell culture}

The ES cells were isolated from blastocysts. The feeder-free culture was performed on plates pre-coated with laminin (Sigma). ES cell differentiation was induced by LIF withdrawal and $0.27 \mu \mathrm{M}$ retinoic acid treatment for $5 \mathrm{~d}$. All of the above cells were cultivated at $37^{\circ} \mathrm{C}$ in $5 \% \mathrm{CO}_{2}(\mathrm{v} / \mathrm{v})$.

\section{Plasmids and antibodies}

Rabbit anti-H2A.B1 and H2A.B2 antibodies were raised against $\mathrm{KLH}$ conjugated peptides of the mouse H2A.B1 N terminus (PRNTENCLQRSSGHRQHH), mouse H2A.B $2 \mathrm{~N}$ terminus (PRNRENCLRESGRRRR), and the common mouse H2A.B C terminus (DVFQFITISQVAPAHR). Anti-HA antibody was purchased from Sigma. Anti- H4 antibody was purchased from Upstate. Anti$5 \mathrm{mC}$ antibody (162 33 D3) was purchased from Calbiochem. AntiRNA Pol II (8WG16) and Pol II pSer2 (H5) antibodies were purchased from Covance.

\section{Cell lysis, immunoprecipitation, and Western blotting}

Cells were lysed with NETN100 buffer (0.5\% NP-40, $50 \mathrm{mM}$ Tris$\mathrm{HCl}, \mathrm{pH}$ 8.0, $2 \mathrm{mM}$ EDTA, and $100 \mathrm{mM} \mathrm{NaCl}$ ) unless specified. The insoluble pellets (chromatin fractions) were resuspended in $0.2 \mathrm{M}$ $\mathrm{HCl}$. The supernatant was neutralized with $1 \mathrm{M}$ Tris- $\mathrm{HCl}(\mathrm{pH} 8.5)$ for further analysis. Immunoprecipitation and Western blotting were performed following standard protocols.

\section{ChIP sequencing}

The ChIP assay was described above. Irrelevant rabbit IgG was used as the control in ChIP. Mouse ES cells were cross-linked with formaldehyde. The chromatin was isolated from $1 \times 10^{8}$ cells and fragmented to 200 to $500 \mathrm{bp}$ by sonication. DNA was repaired to blunt ends by T4 DNA polymerase and phosphorylated with T4 polynucleotide kinase using the END-IT kit (Epicentre). A single " $\mathrm{A}$ " base was added to the 3 ' end with Klenow. Double-stranded adaptors were ligated to the fragments with DNA ligase. Ligation products between 200 and 600 bp were gel-purified in a $2 \%$ agarose gel to remove unligated adaptors and subjected to 20 PCR cycles. Completed libraries were quantified with PicoGreen (Life Technologies) staining.

\section{Chromatin immunoprecipitation and quantitative-PCR}

Chromatin immunoprecipitation (ChIP) was performed using a ChIP assay kit (Millipore) following the manufacturer's instructions. For each ChIP, $1 \times 10^{6}$ cells were used. Anti-H2A.B antibody was used for ChIP assays. For the analysis of gene expression, total RNA was extracted with TRIzol (Invitrogen), and cDNA was generated using SuperScript III Reverse Transcriptase (Invitrogen). RT-PCR with $G A P D H$-specific primer was used as a control. Quantitative-PCR (qPCR) was performed using Power SYBR green PCR master mix in 7300 real-time PCR systems (Applied Biosystems). The primers are listed in Supplemental Table S2. The mean value was calculated by three or four independent experiments.

\section{MeDIP and hMeDIP}

Genomic DNA isolated from mouse ES cell was sonicated to produce random fragments $\sim 200$ to $400 \mathrm{bp}$. Sonicated DNA was

\section{Genome Research}


denatured at $95^{\circ} \mathrm{C}$ for $10 \mathrm{~min}$ and cooled on ice. Denatured DNA was incubated with $1 \mu \mathrm{g}$ anti-5mC or anti-5hmC antibodies at $4^{\circ} \mathrm{C}$ overnight. Antibody-DNA complexes were captured by Protein A/G beads. Immunoprecipitated DNA was purified and subjected to qPCR with indicated primers.

\section{Retrovirus preparation and infection}

The primers for H2A.B shRNA are listed in Supplemental Table S2. Since the targeting sequences existed in both H2A.B1 and H2A.B2, the shRNAs down-regulated both H2A.B1 and H2A.B2. Retrovirus was collected, concentrated, and added to the culture media of ES cells. The infected cells were selected by G418 for $2-4 \mathrm{wk}$ and then used in the following experiments.

\section{Determining the allele-specific deposition}

Genomic DNA associated with H2A.B was collected by ChIP and amplified by PCR. All PCRs except those for region C of Kcnq1 were carried out with Go-Taq DNA polymerase (Promega) using $0.3 \mu \mathrm{M}$ of each primer (Supplemental Table S2) and $0.1 \mu \mathrm{Ci}$ of $\left[{ }^{32} \mathrm{P}\right] \mathrm{dCTP}$. For region C of Kcnq1, Herculase DNA polymerase (Stratagene) was used for the PCR, with $1 \times$ Herculase buffer, $0.3 \mu \mathrm{M}$ of each primer, $0.8 \mathrm{mM}$ deoxynucleoside triphosphates, $4 \%$ dimethyl sulfoxide, and $0.1 \mu \mathrm{Ci}$ of $\left[{ }^{32} \mathrm{P}\right] \mathrm{dCTP}$. The PCR products were sequenced, and single-nucleotide polymorphism was used to determine the allelespecific deposition of H2A.B. Alternatively, the PCR product was digested by restriction enzymes, and different digestion patterns between mus allele and cas allele were used to determine the allelespecific deposition of H2A.B (Supplemental Table S4). The relative band intensities were quantified using ImageQuant (Molecular Dynamics). To determine the allele-specific deposition of H2A.B at the B region of $I g f 2 r$, PCR-SSCP (PCR-based Single-strand Conformation Polymorphism) was performed as previously described (Umlauf et al. 2004). In brief, $10^{7}$ cells were collected and washed in PBS. Nuclei were purified through a sucrose cushion and incubated with MNase to obtain fragments of one to five nucleosomes in length. Approximately $5 \mu \mathrm{g}$ of chromatin were incubated with $2 \mu \mathrm{g}$ of antibody overnight at $4^{\circ} \mathrm{C}$. The antibody chromatin complexes were captured by Protein A sepharose beads. After washing and elution, DNA was extracted from the input chromatin and bound fractions. We analyzed DNA from the ChIP assays by PCR-SSCP using primers listed in Supplemental Table S2.The parental alleles were distinguished by radioactive PCR followed by electrophoretic detection of SSCP polymorphisms.

\section{Data analyses of ChIP sequencing and MeDIP sequencing}

For ChIP-seq analysis, DNA libraries were analyzed by Illumina high-throughput sequencing. The read quality of each sample was determined by FastQC software. After prefiltering the raw data by removing sequenced adapters and low quality reads, the tags were mapped to the mouse genome (assembly $\mathrm{mm} 9$ ) by Bowtie software. Parameter settings were as follows: $-v, 3$ (reported alignments with at most three mismatches), $-5,3$ and $-3,3$ (trim 3 bases from $5^{\prime}$ and $3^{\prime}$ end to remove low-quality bases).

Peak detection was performed using MACS software from the Galaxy browser (Zhang et al. 2008). Sequencing reads from irrelevant IgG in ChIP or MeDIP assays were used as a negative control. The unique peaks obtained from ChIP-seq or MeDIP-seq were matched to the annotated reference genome (mouse $\mathrm{mm} 9$ ) using CisGenome 2.0 (Ji et al. 2008).

To examine the distribution of H2A.B, the whole genome was partitioned into four regions: intragenic region (including the 5' UTR, coding exon, intron, and 3' UTR); upstream region (10 kb upstream of the TSS, including most transcription promoters and other transcriptional elements), distal intergenic region that does not encode any genes, and downstream region (10 kb downstream from the TES) (Fig. 1B). Genes not uniquely mapped to the genome were excluded. To avoid redundancy, only the longest transcript variant of each gene was used to define chromosomal locations of the intragenic region, upstream region, distal intergenic region, and downstream region in Figure 1B and Supplemental Figure S4A.

For gene body plots in Figure 1D, 50 nonoverlapping windows with the average tag number per base were calculated for each gene. Ten kilobases upstream of the TSS and $10 \mathrm{~kb}$ downstream from the TES were divided into windows of $1 \mathrm{~kb}$. We first normalized the enrichment of H2A.B and $5 \mathrm{mC}$ with control IgG. For comparing the localization of H2A.B and $5 \mathrm{mC}$ in the gene body region, we set the relative enrichment of H2A.B and $5 \mathrm{mC}$ at $-10 \mathrm{~kb}$ from the TSS as " 1. "

In Figure 1E, we examined the overlapped gene body regions of H2A.B and $5 \mathrm{mC}$. We clustered the distribution of H2A.B into six groups based on its localization in the gene body. In each gene body, we examined whether $5 \mathrm{mC}$ localizes close to H2A.B. In Figure $1 \mathrm{~F}$, we summarized the results from six different cluster analyses in Figure 1E. The relative accumulation of tags around the indicated region in Figure $1 \mathrm{~F}$ was performed using seqMINER software (Ye et al. 2011). H2A.B-enriched regions were used as the reference. Tag densities from each ChIP (MeDIP)-seq were collected within a window of $10 \mathrm{~kb}$ around the reference coordinates. The tag density of H2A.B ( $y$-axis) in a 200-bp window was normalized to " 1 " at the peak region. The tag density of $5 \mathrm{mC}$ in a $200-\mathrm{bp}$ window was normalized with the relative tag density of H2A.B.

More detailed data analyses of ChIP sequencing and MeDIP sequencing are included in the Supplemental Material.

\section{Bisulfite conversion}

Genomic DNA was treated with the bisulfite conversion reagent of the EZ DNA Methylation kit (Zymo Research). After PCR amplification using primer sets that hybridize both methylated and unmethylated clones, PCR products were subcloned with the TOPO TA Cloning kit (Invitrogen) and subjected to direct sequencing.

\section{Affymetrix microarray analysis}

The microarray study was carried out using Mouse Genome 430 2.0 GeneChip arrays (Affymetrix). Total RNA was extracted from cells with the RNeasy kit including DNase digestion (Qiagen). Biotinlabeled cRNA was obtained from $3 \mu \mathrm{g}$ of total RNA with the GeneChip One-Cycle labeling kit (Affymetrix). Fifteen micrograms of cRNA were fragmented and hybridized to Affymetrix 4302.0 GeneChip arrays at $45^{\circ} \mathrm{C}$ for $16 \mathrm{~h}$. DNA chips were washed, stained, and scanned using an Affymetrix Fluidics device and a GCS3000 scanner, and the images obtained were analyzed using the GCOS software. The results are shown in Supplemental Table S5. The expression value of each transcript is shown in $\log _{2}$ scale.

\section{Data access}

Gene expression and sequencing data have been submitted to the NCBI Gene Expression Omnibus (GEO; http://www.ncbi.nlm. nih.gov/geo/) under accession number GSE49294.

\section{Acknowledgments}

We thank Drs. Yali Dou and Yifan Liu for suggestions and Linyu Lu and Henry Kuang for proofreading. This work was supported by 
the National Institutes of Health (CA132755 and CA130899 to X.Y.), the University of Michigan Cancer Center, and GI Peptide Research Center. X.Y. is a recipient of the Era of Hope Scholar Award from the Department of Defense.

\section{References}

Ahmad K, Henikoff S. 2002. The histone variant H3.3 marks active chromatin by replication-independent nucleosome assembly. Mol Cell 9: 1191-1200.

Anamika K, Krebs AR, Thompson J, Poch O, Devys D, Tora L. 2010. Lessons from genome-wide studies: An integrated definition of the coactivator function of histone acetyl transferases. Epigenetics Chromatin 3: 18.

Andrews AJ, Luger K. 2011. Nucleosome structure(s) and stability: Variations on a theme. Annu Rev Biophys 40: 99-117.

Angelov D, Verdel A, An W, Bondarenko V, Hans F, Doyen CM, Studitsky VM, Hamiche A, Roeder RG, Bouvet P, et al. 2004. SWI/SNF remodeling and p300-dependent transcription of histone variant $\mathrm{H} 2 \mathrm{ABbd}$ nucleosomal arrays. EMBO J 23: 3815-3824.

Ausio J. 2006. Histone variants-the structure behind the function. Brief Funct Genomics Proteomics 5: 228-243.

Bao Y, Konesky K, Park YJ, Rosu S, Dyer PN, Rangasamy D, Tremethick DJ, Laybourn PJ, Luger K. 2004. Nucleosomes containing the histone variant H2A.Bbd organize only 118 base pairs of DNA. EMBO J 23: 3314 3324.

Berger SL. 2002. Histone modifications in transcriptional regulation. Curr Opin Genet Dev 12: 142-148.

Braidotti G, Baubec T, Pauler F, Seidl C, Smrzka O, Stricker S, Yotova I, Barlow DP. 2004. The Air noncoding RNA: An imprinted cis-silencing transcript. Cold Spring Harb Symp Quant Biol 69: 55-66.

Burden AF, Manley NC, Clark AD, Gartler SM, Laird CD, Hansen RS. 2005. Hemimethylation and non-CpG methylation levels in a promoter region of human LINE-1 (L1) repeated elements. J Biol Chem 280: 14413-14419.

Campos EI, Reinberg D. 2009. Histones: Annotating chromatin. Annu Rev Genet 43: 559-599.

Cao R, Tsukada Y, Zhang Y. 2005. Role of Bmi-1 and Ring1A in H2A ubiquitylation and Hox gene silencing. Mol Cell 20: $845-854$.

Chadwick BP, Willard HF. 2001. A novel chromatin protein, distantly related to histone $\mathrm{H} 2 \mathrm{~A}$, is largely excluded from the inactive $\mathrm{X}$ chromosome. J Cell Biol 152: 375-384.

Daury L, Chailleux C, Bonvallet J, Trouche D. 2006. Histone H3.3 deposition at E2F-regulated genes is linked to transcription. EMBO Rep 7: 66-71.

Dawson B, Trapp RG. 2004. Basic \& clinical biostatistics. Lange Medical Books-McGraw-Hill, New York.

Doyen CM, Montel F, Gautier T, Menoni H, Claudet C, Delacour-Larose M, Angelov D, Hamiche A, Bednar J, Faivre-Moskalenko C, et al. 2006. Dissection of the unusual structural and functional properties of the variant H2A.Bbd nucleosome. EMBO J 25: 4234-4244.

Ehrlich M, Gama-Sosa MA, Huang LH, Midgett RM, Kuo KC, McCune RA, Gehrke C. 1982. Amount and distribution of 5-methylcytosine in human DNA from different types of tissues of cells. Nucleic Acids Res 10: 2709-2721.

Eirin-Lopez JM, Ishibashi T, Ausio J. 2008. H2A.Bbd: A quickly evolving hypervariable mammalian histone that destabilizes nucleosomes in an acetylation-independent way. FASEB J 22: 316-326.

Ficz G, Branco MR, Seisenberger S, Santos F, Krueger F, Hore TA, Marques CJ, Andrews S, Reik W. 2011. Dynamic regulation of 5-hydroxymethylcytosine in mouse ES cells and during differentiation. Nature 473: 398-402.

Gautier T, Abbott DW, Molla A, Verdel A, Ausio J, Dimitrov S. 2004. Histone variant $\mathrm{H} 2 \mathrm{ABbd}$ confers lower stability to the nucleosome. EMBO Rep $\mathbf{5}$ $715-720$.

Gonzalez-Romero R, Mendez J, Ausio J, Eirin-Lopez JM. 2008. Quickly evolving histones, nucleosome stability and chromatin folding: All about histone H2A.Bbd. Gene 413: 1-7.

Grandjean V, Yaman R, Cuzin F, Rassoulzadegan M. 2007. Inheritance of an epigenetic mark: The CpG DNA methyltransferase 1 is required for de novo establishment of a complex pattern of non-CpG methylation. PLOS ONE 2: e1136.

Green K, Lewis A, Dawson C, Dean W, Reinhart B, Chaillet JR, Reik W. 2007. A developmental window of opportunity for imprinted gene silencing mediated by DNA methylation and the Kcnq1ot1 noncoding RNA. Mamm Genome 18: 32-42.

Grewal SI, Moazed D. 2003. Heterochromatin and epigenetic control of gene expression. Science 301: 798-802.

Haines TR, Rodenhiser DI, Ainsworth PJ. 2001. Allele-specific non-CpG methylation of the Nf1 gene during early mouse development. Dev Biol 240: $585-598$.
Hellman A, Chess A. 2007. Gene body-specific methylation on the active X chromosome. Science 315: 1141-1143.

Hodl M, Basler K. 2009. Transcription in the absence of histone H3.3. Curr Biol 19: 1221-1226.

Ishibashi T, Li A, Eirin-Lopez JM, Zhao M, Missiaen K, Abbott DW, Meistrich M, Hendzel MJ, Ausio J. 2010. H2A.Bbd: An X-chromosome-encoded histone involved in mammalian spermiogenesis. Nucleic Acids Res 38: 1780-1789.

Ji H, Jiang H, Ma W, Johnson DS, Myers RM, Wong WH. 2008. An integrated software system for analyzing ChIP-chip and ChIP-seq data. Nat Biotechnol 26: 1293-1300.

Jones PA. 2012. Functions of DNA methylation: Islands, start sites, gene bodies and beyond. Nat Rev Genet 13: 484-492.

Jullien J, Astrand C, Szenker E, Garrett N, Almouzni G, Gurdon JB. 2012. HIRA dependent H3.3 deposition is required for transcriptional reprogramming following nuclear transfer to Xenopus oocytes. Epigenetics Chromatin 5: 17.

Kim M, Park YK, Kang TW, Lee SH, Rhee YH, Park JL, Kim HJ, Lee D, Kim SY, Kim YS. 2013. Dynamic changes in DNA methylation and hydroxymethylation when hES cells undergo differentiation toward a neuronal lineage. Hum Mol Genet 23: 657-667.

Klenov MS, Gvozdev VA. 2005. Heterochromatin formation: Role of short RNAs and DNA methylation. Biochemistry (Mosc) 70: 1187-1198.

Kozlenkov A, Roussos P, Timashpolsky A, Barbu M, Rudchenko S, Bibikova M, Klotzle B, Byne W, Lyddon R, Di Narzo AF, et al. 2013. Differences in DNA methylation between human neuronal and glial cells are concentrated in enhancers and non-CpG sites. Nucleic Acids Res 42: 109-127.

Latos PA, Stricker SH, Steenpass L, Pauler FM, Huang R, Senergin BH, Regha $\mathrm{K}$, Koerner MV, Warczok KE, Unger C, et al. 2009. An in vitro ES cell imprinting model shows that imprinted expression of the Igf2r gene arises from an allele-specific expression bias. Development 136: 437-448.

Lee MP, DeBaun MR, Mitsuya K, Galonek HL, Brandenburg S, Oshimura M, Feinberg AP. 1999. Loss of imprinting of a paternally expressed transcript, with antisense orientation to KVLQT1, occurs frequently in Beckwith-Wiedemann syndrome and is independent of insulin-like growth factor II imprinting. Proc Natl Acad Sci 96: 5203-5208.

Luger K, Mader AW, Richmond RK, Sargent DF, Richmond TJ. 1997. Crystal structure of the nucleosome core particle at 2.8 A resolution. Nature 389: 251-260.

Mancini-Dinardo D, Steele SJ, Levorse JM, Ingram RS, Tilghman SM. 2006 Elongation of the Kcnq1ot1 transcript is required for genomic imprinting of neighboring genes. Genes Dev 20: 1268-1282.

McCormick SM, Eskin SG, McIntire LV, Teng CL, Lu CM, Russell CG, Chittur KK. 2001. DNA microarray reveals changes in gene expression of shear stressed human umbilical vein endothelial cells. Proc Natl Acad Sci 98: 8955-8960.

Mito Y, Henikoff JG, Henikoff S. 2005. Genome-scale profiling of histone H3.3 replacement patterns. Nat Genet 37: 1090-1097.

Mitsuya K, Meguro M, Lee MP, Katoh M, Schulz TC, Kugoh H, Yoshida MA, Niikawa N, Feinberg AP, Oshimura M. 1999. LIT1, an imprinted antisense RNA in the human KvLQT1 locus identified by screening for differentially expressed transcripts using monochromosomal hybrids. Hum Mol Genet 8: 1209-1217.

Pastor WA, Pape UJ, Huang Y, Henderson HR, Lister R, Ko M, McLoughlin EM, Brudno Y, Mahapatra S, Kapranov P, et al. 2011. Genome-wide mapping of 5-hydroxymethylcytosine in embryonic stem cells. Nature 473: 394-397.

Phatnani HP, Greenleaf AL. 2006. Phosphorylation and functions of the RNA polymerase II CTD. Genes Dev 20: 2922-2936.

Ramsahoye BH, Biniszkiewicz D, Lyko F, Clark V, Bird AP, Jaenisch R. 2000. Non-CpG methylation is prevalent in embryonic stem cells and may be mediated by DNA methyltransferase 3a. Proc Natl Acad Sci 97: 52375242 .

Risinger JI, Maxwell GL, Chandramouli GV, Jazaeri A, Aprelikova O, Patterson T, Berchuck A, Barrett JC. 2003. Microarray analysis reveals distinct gene expression profiles among different histologic types of endometrial cancer. Cancer Res 63: 6-11.

Schwartz BE, Ahmad K. 2005. Transcriptional activation triggers deposition and removal of the histone variant H3.3. Genes Dev 19: 804-814.

Shin DJ, Joshi P, Hong SH, Mosure K, Shin DG, Osborne TF. 2012. Genomewide analysis of FoxO1 binding in hepatic chromatin: Potential involvement of FoxO1 in linking retinoid signaling to hepatic gluconeogenesis. Nucleic Acids Res 40: 11499-11509.

Soboleva TA, Nekrasov M, Pahwa A, Williams R, Huttley GA, Tremethick DJ. 2012. A unique H2A histone variant occupies the transcriptional start site of active genes. Nat Struct Mol Biol 19: 25-30.

Tahiliani M, Koh KP, Shen Y, Pastor WA, Bandukwala H, Brudno Y, Agarwal S, Iyer LM, Liu DR, Aravind L, et al. 2009. Conversion of 5-methylcytosine to 5-hydroxymethylcytosine in mammalian DNA by MLL partner TET1. Science 324: 930-935.

\section{Genome Research}


Tan L, Xiong L, Xu W, Wu F, Huang N, Xu Y, Kong L, Zheng L, Schwartz L, Shi Y, et al. 2013. Genome-wide comparison of DNA hydroxymethylation in mouse embryonic stem cells and neural progenitor cells by a new comparative hMeDIP-seq method. Nucleic Acids Res 41: e84.

Thakur N, Tiwari VK, Thomassin H, Pandey RR, Kanduri M, Gondor A, Grange T, Ohlsson R, Kanduri C. 2004. An antisense RNA regulates the bidirectional silencing property of the Kcnq1 imprinting control region. Mol Cell Biol 24: 7855-7862.

Tolstorukov MY, Goldman JA, Gilbert C, Ogryzko V, Kingston RE, Park PJ. 2012. Histone variant H2A.Bbd is associated with active transcription and mRNA processing in human cells. Mol Cell 47: 596-607.

Tucker KL. 2001. Methylated cytosine and the brain: A new base for neuroscience. Neuron 30: 649-652.

Umlauf D, Goto Y, Cao R, Cerqueira F, Wagschal A, Zhang Y, Feil R. 2004. Imprinting along the Kcnq1 domain on mouse chromosome 7 involves repressive histone methylation and recruitment of Polycomb group complexes. Nat Genet 36: 1296-1300.

Wang C, Sandling JK, Hagberg N, Berggren O, Sigurdsson S, Karlberg O, Ronnblom L, Eloranta ML, Syvanen AC. 2013. Genome-wide profiling of target genes for the systemic lupus erythematosus-associated transcription factors IRF5 and STAT4. Ann Rheum Dis 72: 96-103.

Weaver JR, Susiarjo M, Bartolomei MS. 2009. Imprinting and epigenetic changes in the early embryo. Mamm Genome 20: 532-543.
Wu H, D'Alessio AC, Ito S, Wang Z, Cui K, Zhao K, Sun YE, Zhang Y. 2011. Genome-wide analysis of 5-hydroxymethylcytosine distribution reveals its dual function in transcriptional regulation in mouse embryonic stem cells. Genes Dev 25: 679-684.

Yan J, Zierath JR, Barres R. 2011. Evidence for non-CpG methylation in mammals. Exp Cell Res 317: 2555-2561.

Ye T, Krebs AR, Choukrallah MA, Keime C, Plewniak F, Davidson I, Tora L. 2011. seqMINER: An integrated ChIP-seq data interpretation platform. Nucleic Acids Res 39: e35.

Zhang Y, Liu T, Meyer CA, Eeckhoute J, Johnson DS, Bernstein BE, Nusbaum C, Myers RM, Brown M, Li W, et al. 2008. Model-based analysis of ChIPSeq (MACS). Genome Biol 9: R137.

Zhou J, Fan JY, Rangasamy D, Tremethick DJ. 2007. The nucleosome surface regulates chromatin compaction and couples it with transcriptional repression. Nat Struct Mol Biol 14: 1070-1076.

Ziller MJ, Muller F, Liao J, Zhang Y, Gu H, Bock C, Boyle P, Epstein CB, Bernstein BE, Lengauer T, et al. 2011. Genomic distribution and intersample variation of non-CpG methylation across human cell types. PLoS Genet 7: e1002389.

Received February 27, 2013; accepted in revised form January 7, 2014. 


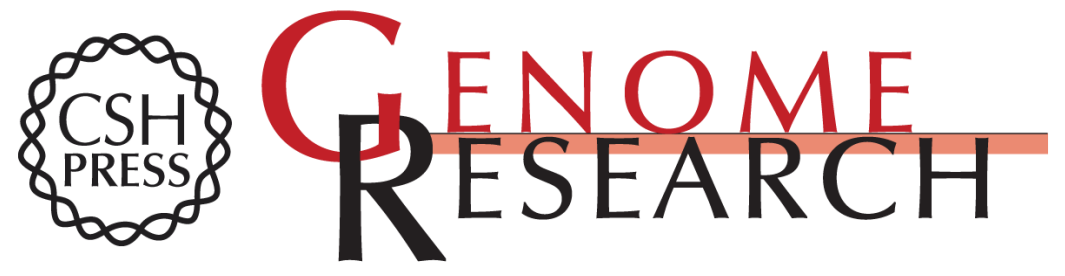

\section{H2A.B facilitates transcription elongation at methylated CpG loci}

Yibin Chen, Qiang Chen, Richard C. McEachin, et al.

Genome Res. 2014 24: 570-579 originally published online January 8, 2014

Access the most recent version at doi:10.1101/gr.156877.113

\section{Supplemental http://genome.cshlp.org/content/suppl/2014/01/15/gr.156877.113.DC1 \\ Material}

References This article cites 60 articles, 22 of which can be accessed free at: http://genome.cshlp.org/content/24/4/570.full.html\#ref-list-1

Creative This article is distributed exclusively by Cold Spring Harbor Laboratory Press for the Commons License first six months after the full-issue publication date (see http://genome.cshlp.org/site/misc/terms.xhtml). After six months, it is available under a Creative Commons License (Attribution-NonCommercial 3.0 Unported), as described at http://creativecommons.org/licenses/by-nc/3.0/.

Email Alerting Receive free email alerts when new articles cite this article - sign up in the box at the Service top right corner of the article or click here.

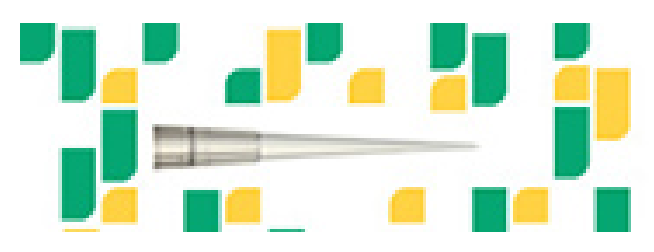

Focused on your science.

To subscribe to Genome Research go to:

https://genome.cshlp.org/subscriptions 